\title{
Initial T Wave Morphology in the Chest Leads in Patients Presenting with Anterior ST-Segment Elevation Myocardial Infarction and its Correlation with Spontaneous Reperfusion of the Left Anterior Descending Coronary Artery
}

\author{
Dalia Azab, Mohamed Elsayed Zahran'1, Ahmed Elmahmoudy' \\ Department of Cardiology, National Heart Institute, Giza, ${ }^{1}$ Department of Cardiology, Ainshams University, Cairo, Egypt \\ ORCID: \\ Mohamed Elsayed Zahran: https://orcid.org/0000000168431374
}

\section{Abstract}

Background: T wave inversion in leads with ST-segment elevation after reperfusion therapy is considered a sign of reperfusion. However, the significance of T wave inversion on presentation before the initiation of reperfusion therapy is unclear. Aim of the Work: The current study aimed to assess whether the initial T wave morphology in the electrocardiographic (ECG) at presentation can predict patency of the left anterior descending artery (LAD) in patients with acute anterior ST segment elevation myocardial infarction (STEMI) before undergoing primary percutaneous coronary interventions (PCIs). Methods: This study included ninety patients who presented to the emergency department with acute anterior ST-elevation MI. We excluded patients with bundle branch block, postcoronary artery bypass grafting patients, patients with paced rhythm, and patients who received thrombolytic therapy. The T wave morphology in the 2 leads with maximal ST-segment elevation on the presenting ECG was identified as one of the three morphologies, positive $\mathrm{T}$ waves ( $\mathrm{T}+$; initial positive deflection $\geq 0.5 \mathrm{~mm}$ above the isoelectric line), biphasic $\mathrm{T}$ waves ( $\mathrm{T}+/-$; where the $\mathrm{T}$ wave initially showed a positive deflection above the ST segment afterward followed by a negative deflection $\geq 0.5 \mathrm{~mm}$ below the isoelectric line), and negative $T$ waves ( $\mathrm{T}-$; where the $\mathrm{T}$ wave initially showed a negative deflection $\geq 0.5 \mathrm{~mm}$ below the isoelectric line without showing any initial positive deflection). Then, according to the results of the initial angiography, patients were classified into spontaneous reperfusion (SR) (those with thrombolysis in MI [TIMI] II or TIMI III flow in the infarct-related artery [IRA] prior to intervention) or non-SR (those with TIMI 0 or TIMI I flow in the IRA prior to intervention). Results: Ninety consecutive patients (77 males and 13 females) presented by STEMI and treated by primary PCI at cath lab of Ainshams University Hospitals (a 24/7 tertiary referral center for primary PCI) between January 2015 and March 2016 were included in this study, of which 40 patients $(44.4 \%)$ had positive $\mathrm{T}$ waves $(\mathrm{T}+), 34$ patients $(37.8 \%)$ had negative $\mathrm{T}$ waves $(\mathrm{T}-)$, and 16 patients $(17.8 \%)$ had biphasic $\mathrm{T}$ waves $(\mathrm{T}+/-)$. Initial angiogram showed that 18 patients had SR and 72 patients had no SR. With regard to T wave morphology, negative T waves were significantly present in SR group $(66.7 \%$ vs. $30.6 \%, P=0.004)$, whereas positive T waves were predominantly present in non-SR (50\% vs. $22.2 \%, P=0.033)$. Conclusions: For SR of LAD in anterior STEMI patients, prior to primary PCI, T wave inversion had a good sensitivity of $66.7 \%$, a specificity of $69.4 \%$, and a good negative predictive value of $89.29 \%$.

Keywords: Anterior ST-segment elevation myocardial infarction, spontaneous reperfusion, T wave

\section{INTRODUCTION}

Myocardial infarction (MI) can be diagnosed clinically by chest pain and other characteristics, electrocardiographic (ECG) changes, elevated levels of biomarkers of myocardial necrosis, and by imaging, or

Submission: 01-Jan-19 Revision: 19-Feb-19 Accepted: 26-Feb-19

\section{Access this article online}

Quick Response Code:

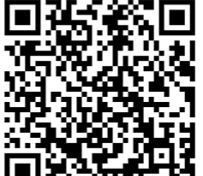

Website:

http://www.ijcva.com

DOI:

10.4103/IJCA.IJCA_1_19
Address for correspondence: Dr. Mohamed Elsayed Zahran, Doctor Mohamed Zahran's Cardiology Clinic, 3 Abdelazeem Awadallah Street, Higaz Square, Heliopolis, Postal Code: 11786, Cairo, Egypt. Ainshams University Hospitals, Cardiology Department, Abbasia Square, Ramses Street, Postal Code: 11517, Cairo, Egypt. E-mail: zahrancardiology@yahoo.com

This is an open access journal, and articles are distributed under the terms of the Creative Commons Attribution-NonCommercial-ShareAlike 4.0 License, which allows others to remix, tweak, and build upon the work non-commercially, as long as appropriate credit is given and the new creations are licensed under the identical terms.

For reprints contact: reprints@medknow.com

How to cite this article: Azab D, Zahran ME, Elmahmoudy A. Initial T wave morphology in the chest leads in patients presenting with anterior ST-segment elevation myocardial infarction and its correlation with spontaneous reperfusion of the left anterior descending coronary artery. Int J Cardiovasc Acad 2019;5:52-7. 
finally by pathological identification of myocardial tissue necrosis. ${ }^{[1]}$

The ECG is the primary diagnostic tool used to identify ST-segment elevation MI (STEMI) that is necessary and crucial because it identifies those patients who are candidates for emergent reperfusion therapy. The fourth universal definition of MI, defined by the Joint Committee of American College of Cardiology (ACC) and the European Society of Cardiology (ESC) for the redefinition of MI, is a new, or presumed new, ST segment elevation in 2 or more contiguous leads of at least $2 \mathrm{~mm}$ at the J point in leads $\mathrm{V} 1-\mathrm{V} 3$ or $1 \mathrm{~mm}$ in other leads. ${ }^{[1]}$

Anterior STEMI results from occlusion of the left anterior descending artery (LAD). Anterior MI carries the worst prognosis of all infarct locations, mostly due to larger infarct size. The nomenclature of anterior infarction can be confusing, with multiple different terms used for the various infarction patterns. The different infarct patterns are named according to the leads with maximal ST elevation, septal infarction with the maximal ST elevation in the chest leads V1-2, anterior with the maximal ST elevation in the chest leads V2-5, anteroseptal with the maximal ST elevation in the chest leads V1-4, anterolateral with the maximal ST elevation in the chest leads V3-6, I + aVL, and finally extensive anterior/anterolateral with the maximal ST elevation in the chest leads V1-6, I + aVL. Although these definitions are intuitive, there is often a poor correlation between ECG features and precise infarct location as determined by imaging or autopsy. ${ }^{[2]}$

The current guidelines for the treatment of ST-segment elevation MIs (STEMI) emphasize the importance of shortening the time interval between the occlusion of the infarct-related artery (IRA) and reperfusion to salvage myocardium and minimize infarct size. ${ }^{[3]}$

In patients with ST-elevation MI (STEMI) undergoing primary percutaneous coronary intervention (PCI), a patent IRA on initial angiography was associated with better angiographic results and improved prognosis compared with patients without spontaneous restoration of the blood flow. Little is known about the prevalence, clinical course, and optimal management of patients presenting with clinical signs of spontaneous reperfusion (SR). SR is defined as $\mathrm{a} \geq 70 \%$ reduction in ST elevation and pain severity before the initiation of reperfusion therapy. ${ }^{[2]}$ Although patients with SR of the IRA have a higher incidence of recurrent in-hospital ischemia, on the longer term, they develop smaller MIs and sustained less in-hospital cardiogenic shock, less incidence of heart failure, and less electrical and arrhythmic complications and have a lower 7- and 30-day mortality rates ${ }^{[4]}$ SR remains significantly associated with a lower incidence of the combined end point of 30-day death, heart failure, and recurrent MI. The outcome of patients who were found to have SR is markedly better than those patients without SR who underwent primary PCI, despite the initial conservative management. ${ }^{[5]}$
SR in ST-elevation MI has traditionally been assessed by coronary angiography. The frequency of SR varies widely in prior studies, and the clinical implications in the modern reperfusion era are unclear. ${ }^{[2,4,5]}$

Negative T waves ( $\mathrm{T}-$ ) in the leads with maximum ST-segment elevation early after initiation of reperfusion therapy whether by thrombolysis or by primary PCI have been described as a marker of reperfusion and a good prognostic sign. ${ }^{[4]}$

However, the significance of $\mathrm{T}$ wave inversion on presentation before the initiation of reperfusion therapy and patency of the IRA (thrombolysis in MI [TIMI] flow grades) is unclear. ${ }^{[6]}$

\section{Methods}

We performed a prospective, single-center observational study at Ainshams University Hospitals, a tertiary referral hospital with a 24/7 primary PCI service offered to all incoming STEMI patients. Ninety consecutive patients admitted from February 2014 to December 2015 from the ED with the diagnosis of anterior STEMI in accordance with the ESC guidelines were included. ${ }^{[1]}$ The study protocol was approved by the local Institutional Ethical Committee. This study included ninety patients who presented to the emergency department with acute anterior ST-elevation MI. We excluded patients with bundle branch block, postcoronary artery bypass grafting patients, patients with paced rhythm, and patients who received thrombolytic therapy. The T wave morphology in the 2 leads with maximal ST-segment elevation on the presenting ECG was identified as one of the three morphologies as shown in Figures 1-3, respectively, positive $T$ waves $(T+$; initial positive deflection $\geq 0.5 \mathrm{~mm}$ above the isoelectric line), negative $\mathrm{T}$ waves ( $\mathrm{T}-$; where the $T$ wave initially showed a negative deflection $\geq 0.5 \mathrm{~mm}$ below the isoelectric line without showing any initial positive deflection), and biphasic $\mathrm{T}$ waves $(\mathrm{T}+/-$; where the $\mathrm{T}$ wave initially showed a positive deflection above the ST segment afterward followed by a negative deflection $\geq 0.5 \mathrm{~mm}$ below the isoelectric line). Then, according to the results of the initial diagnostic angiography prior to intervention, patients were classified into SR (those with TIMI II or TIMI III flow in the IRA prior to intervention) or non-SR (those with TIMI 0 or TIMI I flow in the IRA prior to intervention).

All data were summarized and displayed as mean \pm standard deviation for continuous variables and as number (percentage) of patients in each group for categorical variables. The $P$ values for the categorical variables were calculated with the Chi-square test. Continuous variables were compared using the independent sample $t$-test. A two-tailed $P<0.05$ was considered statistically significant for all analyses. All analyses were performed with the SPSS software (SPSS Inc., Chicago, Illinois, USA).

\section{RESULTS}

According to the occurrence of SR found upon the initial coronary angiogram prior to performing any intervention in 


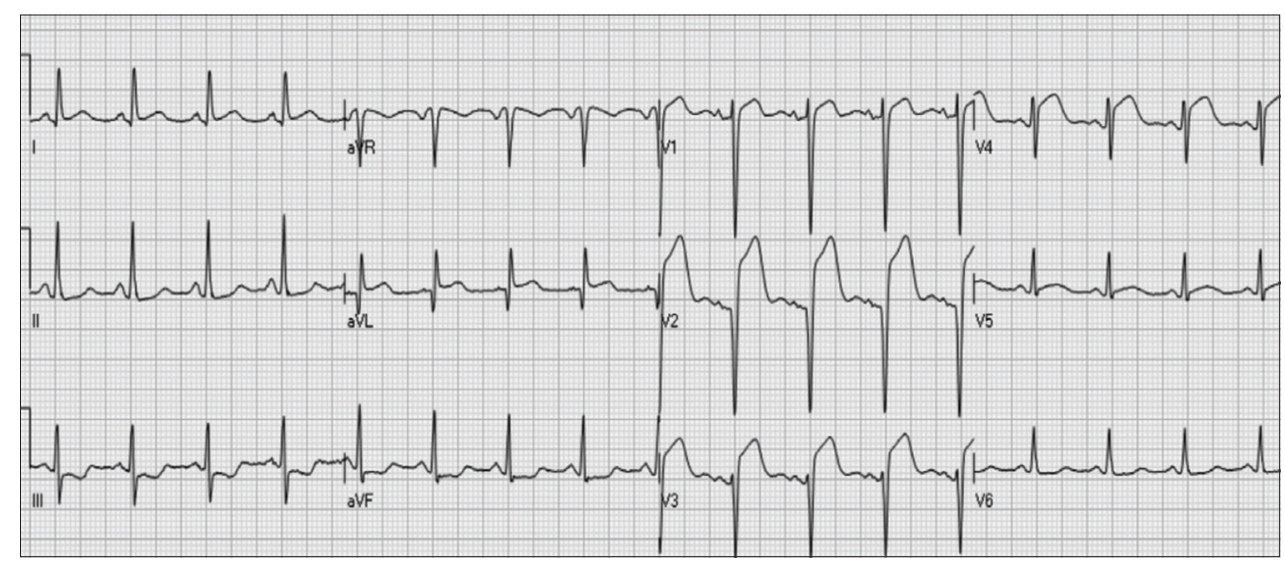

Figure 1: Positive T waves: $T+$; initial positive deflection $\geq 0.5 \mathrm{~mm}$ above the isoelectric line

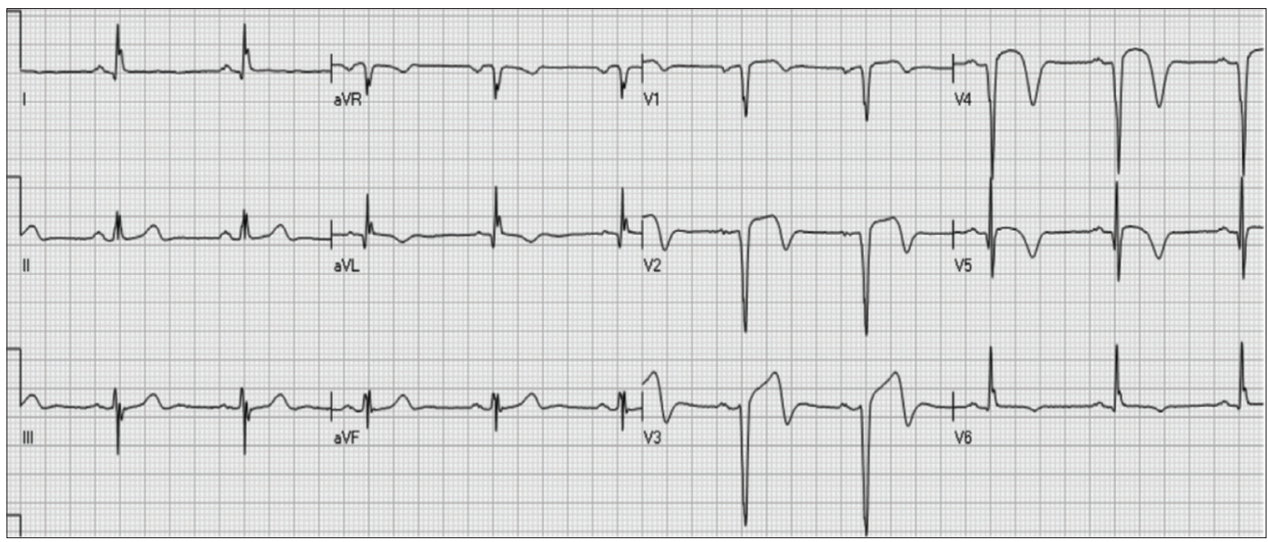

Figure 2: Biphasic T waves: T+/-; initial positive deflection above the ST segment followed by negative deflection $\geq 0.5 \mathrm{~mm}$ below the isoelectric line

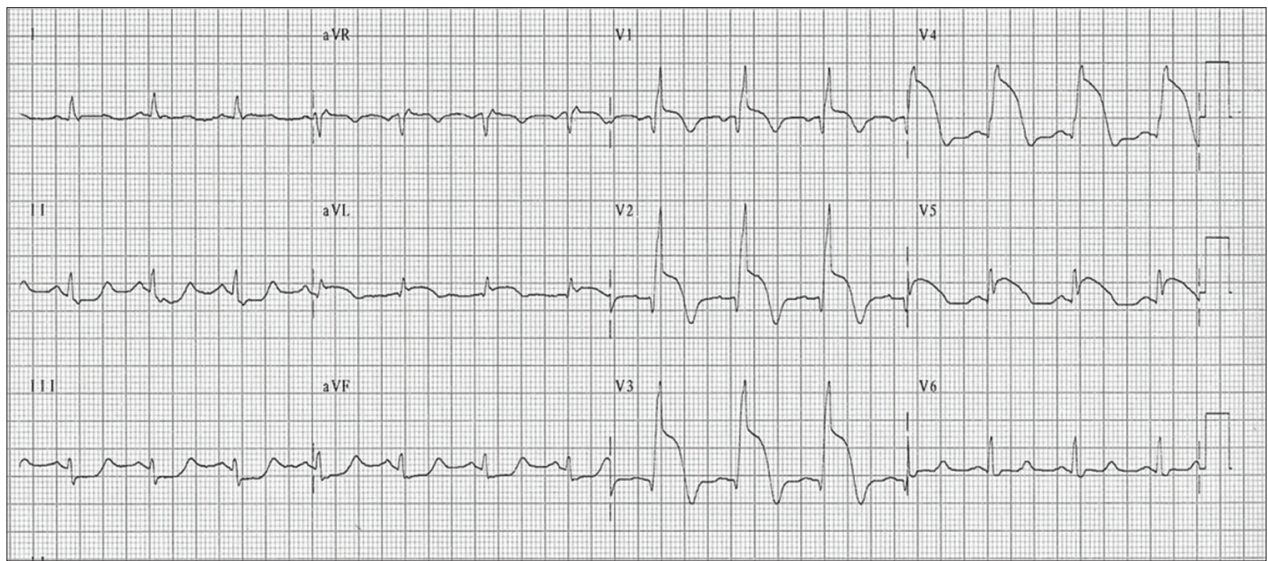

Figure 3: Negative T waves: $T-$; initial negative deflection $\geq 0.5 \mathrm{~mm}$ below the isoelectric line without initial positive deflection

the primary PCI procedure, patients were classified into two groups: SR group, those showing TIMI II or TIMI III flow in the LAD, and non-SR group, those showing TIMI I or TIMI 0 flow in the LAD.

The baseline demographic data are shown in Table 1; patients in the SR group were significantly older than those of the non-SR group $(56.85 \pm 10.23$ years vs. $51.56 \pm 9.30)(P=0.049)$. There was no significant difference between the two groups regarding gender or any of the cardiovascular risk factors.
With regard to $\mathrm{T}$ wave morphology in the presenting ECG prior to revascularization by primary $\mathrm{PCI}$, negative $\mathrm{T}$ waves were significantly present in SR group (66.7\% vs. $30.6 \%$ ), whereas positive $\mathrm{T}$ waves were predominantly present in non-SR group (50\% vs. $22.2 \%$ ) as shown in Table 2 . There was a nonsignificant difference between the two groups regarding the biphasic $\mathrm{T}$ waves $(\mathrm{T}+/ \mathrm{T}-)(11.1 \%$ vs. $19.4 \%)$.

Considering the predictive value of a negative $\mathrm{T}$ wave $\left(\mathrm{T}^{-}\right)$ versus a nonnegative $\mathrm{T}$ wave $(\mathrm{T}+$ or $\mathrm{T}+/-)$, the finding of a 


\begin{tabular}{|c|c|c|c|c|}
\hline & \multirow[t]{2}{*}{ Spontaneous reperfusion $(n=18), n(\%)$} & \multirow[t]{2}{*}{ Nonspontaneous reperfusion $(n=72), n(\%)$} & \multicolumn{2}{|c|}{ Independent $t$-test } \\
\hline & & & $t / \chi^{2 *}$ & $P$ \\
\hline Age, mean \pm SD & $56.85 \pm 10.23$ & $51.56 \pm 9.30$ & 1.997 & 0.049 \\
\hline \multicolumn{5}{|l|}{ Sex } \\
\hline Females & $9(12.5)$ & $4(22.2)$ & $1.101^{*}$ & 0.294 \\
\hline Males & $63(87.5)$ & $14(77.8)$ & & \\
\hline \multicolumn{5}{|l|}{$\mathrm{DM}$} \\
\hline Yes & $30(41.7)$ & $9(50.0)$ & $0.407^{*}$ & 0.523 \\
\hline No & $42(58.3)$ & $9(50.0)$ & & \\
\hline \multicolumn{5}{|l|}{ HTN } \\
\hline Yes & $38(52.8)$ & $11(61.1)$ & $0.403^{*}$ & 0.525 \\
\hline No & $34(47.2)$ & $7(38.9)$ & & \\
\hline \multicolumn{5}{|l|}{ Smoking } \\
\hline Yes & $53(73.6)$ & $10(55.6)$ & $2.235^{*}$ & 0.135 \\
\hline No & $19(26.4)$ & $8(44.4)$ & & \\
\hline \multicolumn{5}{|l|}{ Dyslipidemia } \\
\hline Yes & $53(73.6)$ & $11(61.1)$ & $1.095^{*}$ & 0.295 \\
\hline No & $19(26.4)$ & $7(38.9)$ & & \\
\hline \multicolumn{5}{|l|}{$\mathrm{FH}$} \\
\hline Yes & $35(48.6)$ & 8 (44.4) & $0.100 *$ & 0.752 \\
\hline No & $37(51.4)$ & $10(55.6)$ & & \\
\hline
\end{tabular}

*Significant. SD: Standard deviation, HTN: Hypertension, DM: Diabetes mellitus, FH: Familial hypercholesterolemia

Table 2: Comparison between the two groups regarding $\mathrm{T}$ wave morphology

\begin{tabular}{lccc}
\hline $\begin{array}{l}\text { T wave morphology in leads with maximum ST } \\
\text { elevation }\end{array}$ & Spontaneous reperfusion, $\boldsymbol{n}(\%)$ & Nonspontaneous reperfusion, $\boldsymbol{n}(\%)$ & \multicolumn{1}{c}{ Chi-square test } \\
\cline { 3 - 4 } & & $\chi^{2}$ & $\boldsymbol{P}$ \\
\hline Negative & $12(66.7)$ & $22(30.6)$ & 8.033 \\
Biphasic & $2(11.1)$ & $14(19.4)$ & 0.018 \\
Positive & $4(22.2)$ & $36(50.0)$ & \\
\hline
\end{tabular}

predominantly negative $\mathrm{T}$ wave $(\mathrm{T}-)$ on the initial presenting ECG prior to revascularization by primary PCI had a good sensitivity $(66.7 \%)$ and specificity $(69.4 \%)$ with good negative predictive value $(89.29 \%)$ and a weak positive predictive value $(35.29 \%)$, as shown in Table 3.

\section{Discussion}

This prospective, single-center observational study at Ainshams University Hospitals included a cohort of ninety patients presented by STEMI who underwent primary PCI for revascularization as per guidelines (3). We intended to identify whether the initial $\mathrm{T}$ wave morphology in the ECG at presentation can predict patency of the LAD in patients with acute anterior ST-segment elevation MI (STEMI) before undergoing revascularization by primary PCI.

We found that, regarding the initial $\mathrm{T}$ wave morphology in the presenting ECG prior to revascularization by primary PCI, negative $\mathrm{T}$ waves were significantly present in SR group, whereas positive $\mathrm{T}$ waves were predominantly present in non-SR group. Moreover, the presence of a predominantly negative $\mathrm{T}$ wave $\left(\mathrm{T}^{-}\right)$on the initial presenting
Table 3: The predictive value of a predominantly negative $T$ wave on the initial presenting electrocardiography

\begin{tabular}{lcccc}
\hline & $\begin{array}{c}\text { Spontaneous } \\
\text { reperfusion, } \boldsymbol{n}(\%)\end{array}$ & $\begin{array}{c}\text { Nonspontaneous } \\
\text { reperfusion, } \boldsymbol{n}(\%)\end{array}$ & $\begin{array}{c}\text { Chi-square } \\
\text { test }\end{array}$ \\
\cline { 3 - 5 } & & $22(30.6)$ & 7.988 & 0.004 \\
\hline Negative & $12(66.7)$ & $\chi^{2}$ & $\boldsymbol{P}$ \\
Nonnegative & $6(33.3)$ & $50(69.4)$ & & \\
\hline
\end{tabular}

ECG prior to revascularization by primary PCI had a good sensitivity (66.7\%) and specificity (69.4\%) with good negative predictive value $(89.29 \%)$ for SR.

The diagnostic value of the first ECG to estimate the true disease onset of STEMI has been suggested previously. ${ }^{[2]} \mathrm{T}$ wave inversion and pathological Q waves develop later in the STEMI course, suggesting that more hours have passed the start of the pathological process of STEMI. It is difficult to know accurately the precise time of acute vessel occlusion if the patient cannot differentiate preinfarct angina from the chest pain due to acute STEMI caused by the acute coronary arterial occlusion. ${ }^{[2]}$ 
According to the most recent ESC and ACC guidelines for the treatment of STEMI and also in concordance with the previous versions of the myocardial revascularization guidelines, it is the first priority to shorten the time interval between the occlusion of the IRA and opening the IRA to restore the blood flow and reperfuse the myocardium affected to salvage myocardium and minimize infarct size.$^{[3]}$ This halts the rapid progression of myocardial necrosis because of the presence of ongoing transmural ischemia. Yet, still in some patients presenting with ST-segment elevation, SR, even partially, not necessarily TIMI III, even TIMI II, may abort the progression of myocardial necrosis. ${ }^{[4]}$ The value of stenting in those cases is actually to prevent acute vessel re-occlusion, hence reducing the incidence of reinfarction, rather than immediate myocardial salvage. Hence, in such patients presented by STEMI, if they were stable upon presentation, we may lengthen the door to balloon time beyond the current recommendation of $90 \mathrm{~min}$, similar to the approach for non-STEMI (NSTEMI) cases, where in fact a subset described as those with transient ST segment elevation - but not persistent ST segment elevation - are high-risk NSTEMI mandating intervention within $120 \mathrm{~min}$. ${ }^{[7]}$ Reduction in the severity of symptoms, mainly relief of chest pain, may indicate reperfusion, the presenting symptoms maybe atypical in the elderly, females, patients receiving analgesics, and diabetic patients. ECG changes in such cases probably is a better indicator of reperfusion including ST segment resolution, more than $70 \%$ from the initial ECG at presentation. According to the guidelines, a persistent ST segment elevation (even if there is $>70 \%$ resolution in the magnitude of ST-segment elevation compared with the first ECG), emergency primary PCI is indicated, and the issue of SR is not addressed.

Early after reperfusion therapy, the morphology of the $\mathrm{T}$ waves in the 12-lead surface ECG focusing on the leads with maximum ST-segment elevation has been described as a positive finding indicating coronary artery reperfusion, and this has a good prognostic implication. ${ }^{[6,8]}$ However, it is worth mentioning that $\mathrm{T}$ wave inversion is actually a part of the natural evolution of the ECG changes in STEMI regardless of they received reperfusion therapy or not. ${ }^{[9,10]}$ In these cases, $\mathrm{T}$ wave inversion, in association with pathological $\mathrm{Q}$ waves, represents a marker of an already evolved MI..$^{[9]}$

Herz et al ${ }^{[11]}$ found that, in patients who received tenecteplase for thrombolysis, a predominantly negative $\mathrm{T}$ wave in the 12-lead surface ECG, examined and analyzed in the leads that showed highest ST segment elevation, was associated with better outcomes in the form of reduced mortality in those receiving fibrinolytic therapy in the early golden hours' time window, within the first $2 \mathrm{~h}$ of the start of symptoms, mainly measured from the onset of chest pain. On the other hand, regarding the patients who were treated later, within $2-6 \mathrm{~h}$ after the onset of chest pain, $T$ wave inversion was associated with worse outcomes in the form of increased mortality, a finding that can be attributed to the reduced efficacy of thrombolytic therapy after the first 2 h. ${ }^{[11]}$

\section{Conclusions and Study Limitations}

For SR of LAD in anterior STEMI patients, prior to primary PCI, negative $\mathrm{T}$ waves were significantly present in SR group $(66.7 \%$ vs. $30.6 \%$ with $P=0.004)$, whereas positive $\mathrm{T}$ waves were predominantly present in non-SR group (50\% vs. $22.2 \%$ with $P=0.033$ ). T wave inversion had a good sensitivity of $66.7 \%$, a specificity of $69.4 \%$, and a good negative predictive value of $89.29 \%$.

There are several important limitations of this study. This was a single-center, prospective, and nonrandomized observational study on a small cohort of patients. A long-term follow-up would have added data upon the long-term outcomes of revascularization of primary PCI-treated patients comparing the SR versus the non-SR group.

Further and larger studies are needed to confirm whether the combination of symptom relief; ST-segment resolution $>50 \%$ from the initial magnitude of ST segment elevation; and the T wave morphology whether positive, negative, or biphasic are accurate enough to determine whether there is a difference in the long-term outcomes of primary PCI between these two subsets of patients.

\section{Financial support and sponsorship}

Nil.

\section{Conflicts of interest}

There are no conflicts of interest.

\section{RefEREnCes}

1. Thygesen K, Alpert JS, Jaffe AS, Chaitman BR, Bax JJ, Morrow DA, et al. Fourth universal definition of myocardial infarction (2018). Eur Heart J 2019;40:237-69.

2. Atak R, Ileri M, Senen K, Turhan H, Erbay AR, Basar N, et al. Correlation between infarct-related coronary artery patency and predischarge electrocardiographic patterns in patients with first anterior myocardial infarction who received thrombolytic therapy. Heart Vessels 2004;19:63-7.

3. Ibanez B, James S, Agewall S, Antunes MJ, Bucciarelli-Ducci C, Bueno H, et al. 2017 ESC guidelines for the management of acute myocardial infarction in patients presenting with ST-segment elevation: The task force for the management of acute myocardial infarction in patients presenting with ST-segment elevation of the European Society of Cardiology (ESC). Eur Heart J 2018;39:119-77.

4. Atar S, Barbagelata A, Birnbaum Y. Electrocardiographic markers of reperfusion in ST-elevation myocardial infarction. Cardiol Clin 2006;24:367-76, viii.

5. Doevendans PA, Gorgels AP, van der Zee R, Partouns J, Bär FW, Wellens HJ, et al. Electrocardiographic diagnosis of reperfusion during thrombolytic therapy in acute myocardial infarction. Am J Cardiol 1995;75:1206-10.

6. Sgarbossa EB, Meyer PM, Pinski SL, Pavlovic-Surjancev B, Barbagelata A, Goodman SG, et al. Negative T waves shortly after ST-elevation acute myocardial infarction are a powerful marker for improved survival rate. Am Heart J 2000;140:385-94.

7. Brener SJ, Mehran R, Brodie BR, Guagliumi G, Witzenbichler B, Cristea E, et al. Predictors and implications of coronary infarct artery patency at initial angiography in patients with acute myocardial infarction (from the CADILLAC and HORIZONS-AMI trials). Am J Cardiol 2011;108:918-23.

8. Shimada YJ, Po JR, Kanei Y, Schweitzer P. Prognostic impact of terminal $\mathrm{T}$ wave inversions on presentation in patients with ST-elevation 
myocardial infarction undergoing urgent percutaneous coronary intervention. J Electrocardiol 2013;46:2-7.

9. Eskola MJ, Holmvang L, Nikus KC, Sclarovsky S, Tilsted HH, Huhtala $\mathrm{H}$, et al. The electrocardiographic window of opportunity to treat vs. the different evolving stages of ST-elevation myocardial infarction: Correlation with therapeutic approach, coronary anatomy, and outcome in the DANAMI-2 trial. Eur Heart J 2007;28:2985-91.
10. Sclarovsky S, editor. The evolving acute myocardial infarction. In Electrocardiography of Acute Myocardial Ischaemic Syndromes. London, United Kingdom: Martin Dunitz; 1999. p. 99-122.

11. Herz I, Birnbaum Y, Zlotikamien B, Strasberg B, Sclarovsky S, Chetrit A, et al. The prognostic implications of negative $\mathrm{T}$ waves in the leads with ST segment elevation on admission in acute myocardial infarction. Cardiology 1999;92:121-7. 